\title{
THE USE OF OPTICAL TRANSFER FUNCTION FOR ASSESSING THE QUALITY OF OPTICAL SYSTEMS*
}

\author{
H. TIZIANI \\ Wild Heerbrugg AG, Heerbrugg (Switzerland)
}

(Received August 26, 1976; revised and accepted January 24, 1977)

\begin{abstract}
Tiziani, H., 1978. The use of optical transfer function for assessing the quality of optical systems. Photogrammetria, 34: 45-68.

The optical transfer function (OTF) has proved to be a nighly valuable aid in comparing and evaluating the quality of optical systems. The measuring instrument developed is briefly described. The OTF measurements agreed well with theoretical results obtained from lens data, especially for the modulation transfer function (MTF). With the aid of a model based on experiments, a threshold curve was obtained which enabled the results based on the MTF to be compared with those from conventional resolution tests. To enable workable focusing and quality criteria to be derived, an attempt was made to condense the profuse data resulting from the MTF.
\end{abstract}

\section{INTRODUCTION}

Over the last few years, the image quality of aerial camera lenses has improved enormously, so that today it is astonishing what these lenses can achieve.

In recent years, image quality has received considerable attention, as is apparent from the efforts made to obtain reliable criteria for its assessment. The search for image quality assessment techniques has not been limited to aerial camera lenses, however, but has encompassed other optical systems as well (Baker, 1971; Brock et al., 1972; Consitt and Mandler, 1971; Hopkins and Dutton, 1971; Matsui and Murata, 1971; Norton, 1975; Pouleau and Lafouasse, 1972). Today, several national and international committees are studying the optical transfer function (OTF) and its use in evaluating image quality. In various countries OTF specifications, which are often complex, have already been derived. OTF and MTF (modulation transfer function) have still not been generally accepted as image-evaluation criteria**. There

*Presented Paper, Commission I, 13th International Congress for Photogrammetry, Helsinki, 1976.

**The OTF covers modulation and phase, whereas the MTF does not include phase shifts. 
are two reasons for this: firstly, differences occurred between calculated values when using different computer programs, as well as in their comparison with actual measured results; secondly, experiments carried out on identical optical systems, but at different places and with different test equipment, have sometimes shown different results. Various commissions have already studied the possibilities of standardizing the OTF and especially the measurement criteria (British Standards, DIN) (British Standard BS 4779, 1971; DIN 58185; Welch, 1974). Standardizing the measurement criteria would improve the consistency of results from different laboratories. Especially for wide-angle lenses, results from different laboratories, when using the same lenses, showed differences of about $20-30 \%$ a few years ago. Since then both the measuring procedures and the measurement criteria have been perfected, as is evidenced by the results of the most recent comparative measurements organized by the DIN Standards Committee (DIN = German Industrial Standards). This applies, in particular, to the modulation transfer function (MTF). The phase measurements were not compared. Phase has not often been used in comparison measurements, but could be very useful for the optical designer in evaluating new systems and also for the determination of asymmetries. In addition, a large phase error usually implies a low MTF value; the MTF alone can therefore be useful for workshop tests. For the measurement of distortion, other techniques are applied.

The results of the latest DIN comparison tests are very encouraging for future work in elaborating image quality criteria based on MTF. A few typical results of the latest DIN comparative measurements are described and graphed in the next section of the paper. OTF also offers the optical designer valuable information on the quality of newly calculated systems.

The ISP has long been interested in standardizing the OTF for aerial camera lenses, especially because of the objectivity of this method and the very small time required for the measurements. The results of the Working Group, Commission I, are presented in a comprehensive report by Norton (1975).

\section{RESULTS OF THE SECOND DIN-MTF COMPARISON TEST}

Different comparison tests were carried out recently (Sira Institute report, DIN-report, not yet released for general distribution). The DIN Standards Committee comprises representatives of the optical and electro-optical industry in Germany and Switzerland as well as members from universities. Three comparison tests were undertaken by the DIN Standards Committee and two of these have been completed. With regard to the third test, the lens under investigation has been circulating around various laboratories and the results will be compared soon.

The comparison tests already completed on a particular "Rodenstock" wideangle lens show very satisfactory agreement. Although the measurements were made with different methods and instruments, deviations in the results were usually less than 0.05 (the MTF is normalized to 1.0 for zero spatial frequency). 
The test lenses were manufactured and mounted with the utmost precision. Special attention was also paid to centring the lenses. All technical characteristics of the lenses were known. At the same time, precise test specifications were drawn up in order to carry out the measurements under identical conditions (DIN standards). Typical results of the comparison tests with the wideangle lens $f=90 \mathrm{~mm}, f / 5.6$ are shown in Figs. 1 and 2 , for the wavelength $\lambda=$ $546 \mathrm{~nm}$ and band width $20 \mathrm{~nm}$ for the field angles $w=26.6^{\circ}$ and $45.1^{\circ}$. The appropriate image position was chosen to be at the maximum of the axial MTF at 60 line pairs per mm. Deviations in the results were found to be far smaller than those obtained in previous comparison tests carried out by other groups. The results of measurements from seven laboratories lie within the two plotted curves. It is apparent from the figures that the theoretical results also show discrepancies. The spread of the computed results, obtained by different computer programs, is shown by the vertical lines.

If one compares the results of previous comparison tests, for example the

$f / 5,6 f=90 \mathrm{~mm} ; w=26,6^{\circ}$ tan
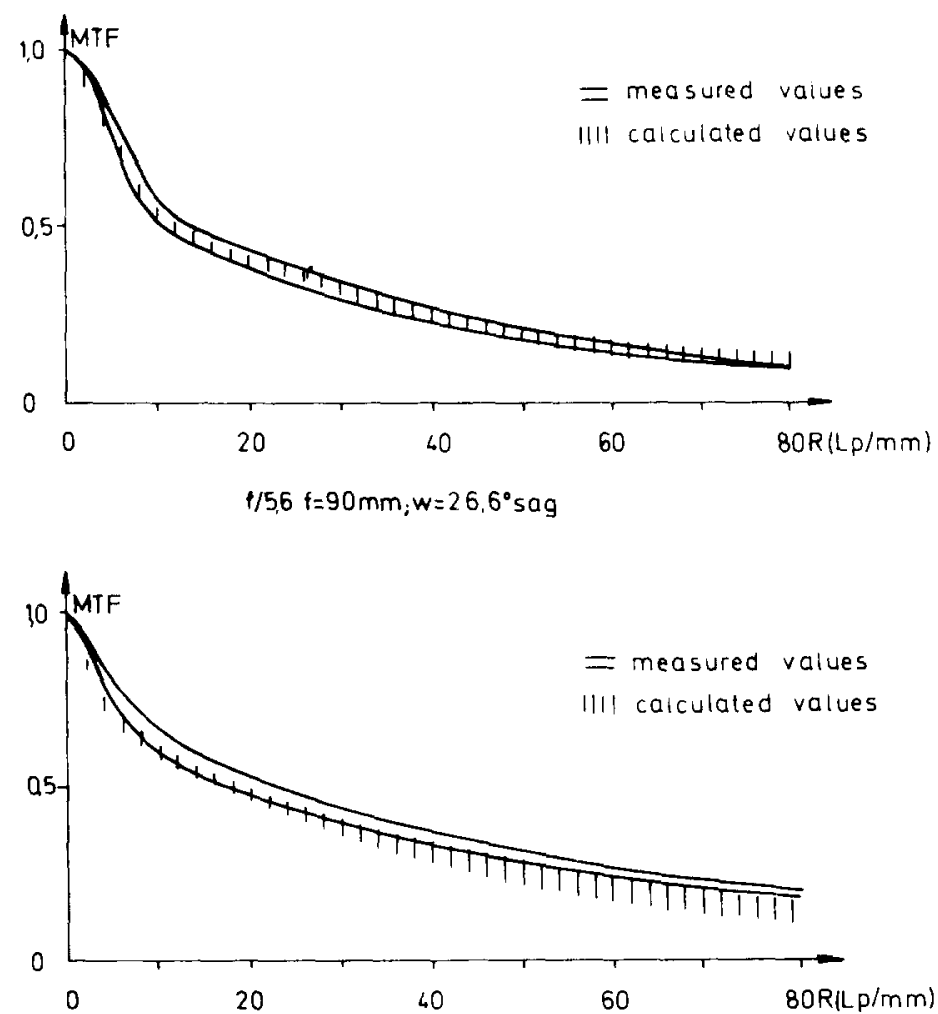

Fig. 1. Results of the comparison test, in conjunction with the DIN Standards Committee, on the Rodenstock lens $f=90 \mathrm{~mm}, f / 5.6$, tangential and sagittal, for field angle $w=26.6^{\circ}$. 
$f / 5,6 f=90 \mathrm{~mm} ; w=45.11^{\circ}$ an
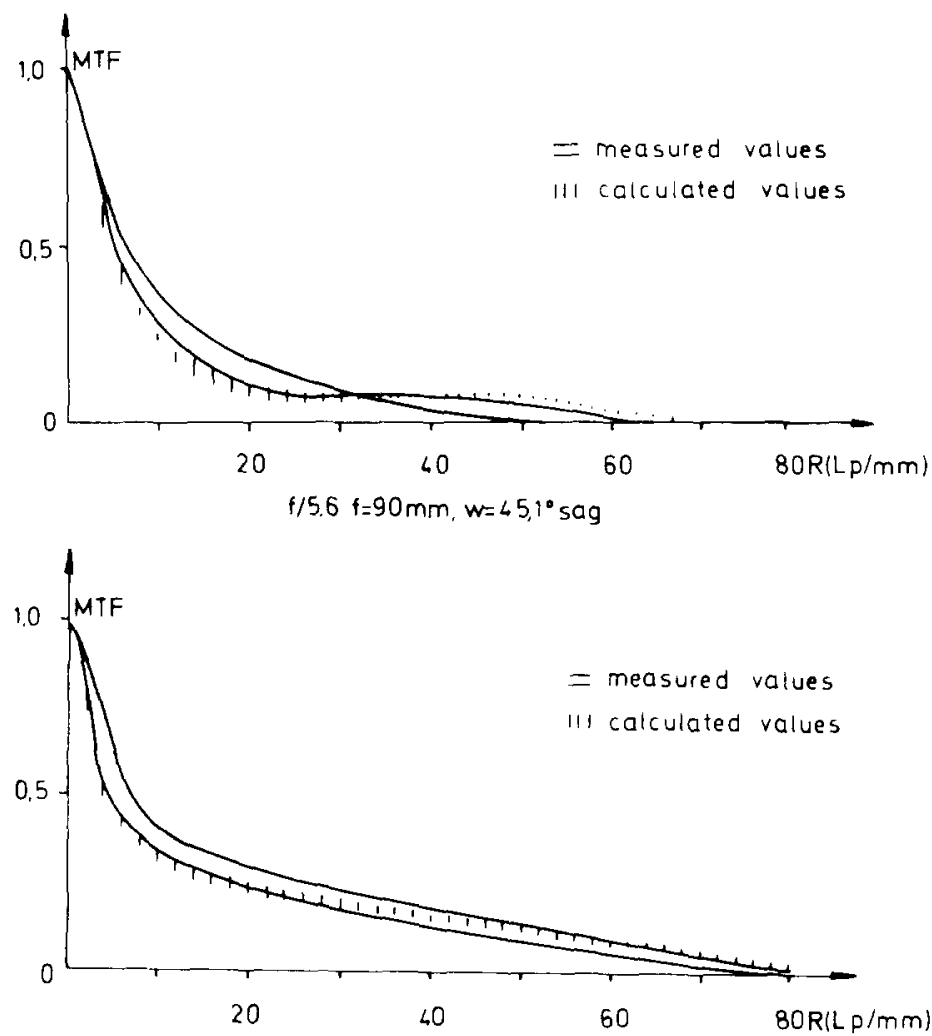

Fig. 2. Results of the comparison test, in conjunction with the DIN Standards Committee, on the Rodenstock lens $f=90 \mathrm{~mm}, f / 5.6$, tangential and sagittal, for field angle $w=45.1^{\circ}$.

one by Hopkins and Dutton (1971) with those of Figs. 1 and 2, one may conclude that the instruments and especially the measuring techniques have been improved considerably. It appears that in the not too distant future, the optical transfer function could well be adopted as a quantitative technique for assessing the quality of the optical system.

\section{EQUIPMENT USED IN MEASURING THE OPTICAL TRANSFER FUNCTION}

For the measurement of the OTF or MTF, different measuring principles are already being used (Murata, 1966). They can be grouped as follows:

(1) scanning the spread function and carrying out the associated Fourier transformation;

(2) interference methods (two-beam interference and holography);

(3) direct Fourier transformation. 
For our purposes, the direct Fourier transformation method proved to be the most suitable measuring technique. The principle will be reviewed briefly. A test grating is reproduced through the test lens and the contrast compared in the image with that of the test object (Fig. 3). According to the Fourier transformation theory every object can be considered to be composed of sinusoidal functions. The optical system consequently acts as a filter for the transfer of fixed frequencies. The principle of the direct Fourier transformation is briefly summarized below. The contrast in the object can be written:

$C_{\text {object }}=\frac{I_{\max }-I_{\min }}{I_{\max }+I_{\min }}=\frac{\beta}{\alpha}$

According to Fig. 3 the contrast in the image for a given spatial frequency can be written as:

$C_{\text {image }}=\frac{\beta^{\prime}}{\alpha}$

The ratio of the contrast in the image to that in the object is the modulation transfer (MTF) for a given spatial frequency:

$\operatorname{MTF}(R)=\frac{C_{\text {image }}(R)}{C_{\text {object }}(R)}=\frac{\beta^{\prime}(R)}{\beta(R)}$

A possible phase error $\theta(R)$ appears as a lateral displacement of the reproduction. The contrast and phase measurement are repeated for various spatial frequencies, and result in the optical transfer function (OTF).

The principle of the measuring equipment we developed and applied is based on a direct Fourier transformation. In effect, the instrument measures the contrast in the image plane for every fixed frequency and compares it with that of the object, the grid. In this paper, phase measurement will not be discussed. The equipment constructed by Wild for measuring the OTF is illustrated schematically in Fig. 4. A continually moving transmission grid is reproduced and scanned by the image slit, i.e., the modulation of the grid reproduction is compared with that of the grid in the object generator. A $150-W$ halogen lamp with diffuser, heat protection filter and suitable colour

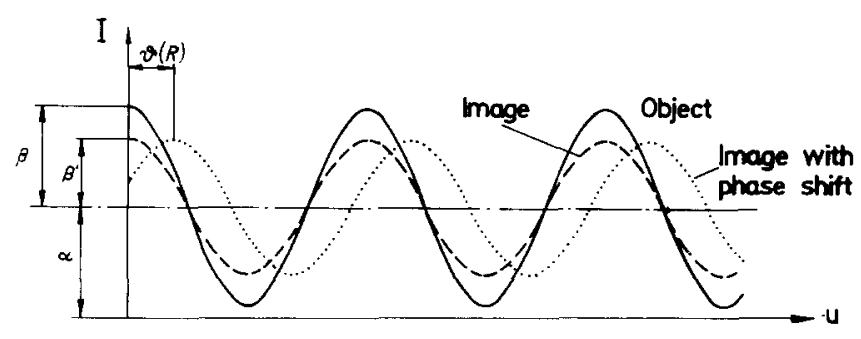

Fig. 3. Principle of the optical transfer function. 


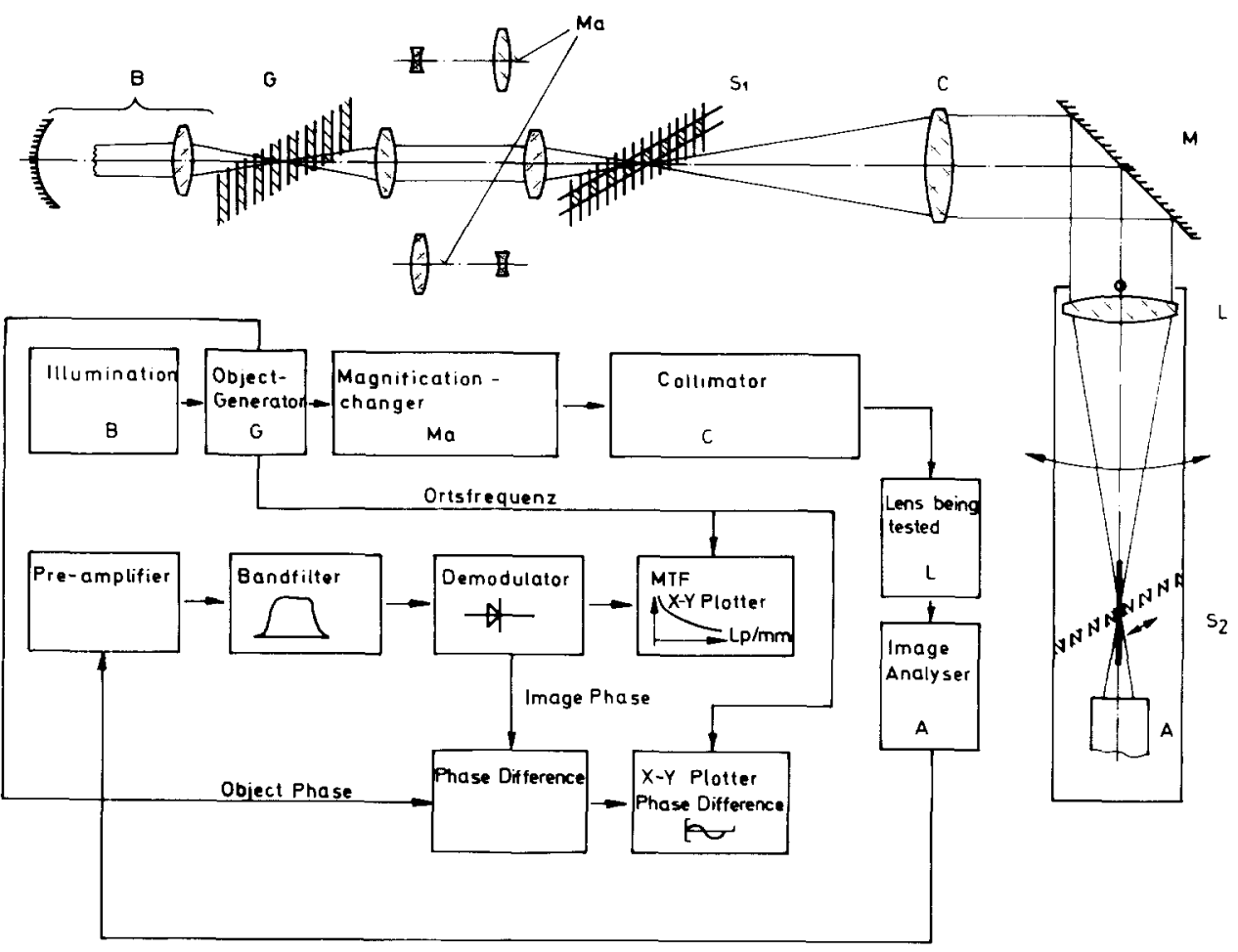

Fig. 4. Principle of the equipment.

filters is used as a light source. The object grid $G$ is a radial grid (Beck: Ealing) (period $10 \mathrm{lp} / \mathrm{mm}$ ). It is used in an arrangement modified from that of the EROS 100. However, the radial grid produces a rectangular transmissivity and not a sinus-shaped one, as a result of the production technique. By electrically filtering the exit signal, the sinus grid transmission is accomplished (band filter for fundamental oscillations of the Fourier Series). The latter is reproduced on the entry slit $S_{1}$ with a magnification changer. The continuously moving radial grid (frequency $=1000 \mathrm{~Hz}$ ) is reproduced on the exit slit $S_{2}$ of the analyser $A$. The change in frequency is achieved by tilting the grid in relation to the entry slit. The period varies with the cosine of the angle of tilt of the grid in relation to slit $S_{1}$. The magnification changer for reproducing the grid on the slit permits five magnifications between $0.4 \times$ and $2.5 \times$.

The collimator $C$ projects the object slit with the reproduction of the grid to infinity. Collimators with a focal length of $f=570 \mathrm{~mm}$ are available along with a superachromatic telescopic lens with $f=2000 \mathrm{~mm}$ at $1000 \mathrm{~mm}$ total length, which has been especially designed by Wild Heerbrugg, Ltd. This lens is extremely well corrected (diffraction limited for $400<\lambda<1000 \mathrm{~nm}$ ). The free diameter is $100 \mathrm{~mm}$. The quality of the collimator was tested by means 
of the Foucault Test Method as well as interferometrically. No significant error was found.

Object and image slits $S_{1}$ and $S_{2}$ were manufactured by vacuum deposition. They can be rapidly interchanged so that, in most cases, the width of the slits can be chosen in order to neglect a slit correction. The selection of the slit width varies from 1 to $50 \mu \mathrm{m}$. Furthermore, the instrument was designed to measure almost to the very edge of the image of any aerial survey lens with the picture frame mounted.

For off-axis image points (up to half of the field angle $w=60^{\circ}$ ) the sliding carriage with the image analyser and test sample is rotated around the entry pupil of the test objective $L$. To achieve this, the carriers are rotated on air cushions. The entire apparatus rests on a stone base plate embedded in sand, the whole being supported by automobile tyres. The inherent frequency of the basic structure is $2 \mathrm{~Hz}$. Special attention was paid to assuring a stable structure, since the equipment was particularly designed for checking wide-angle lenses for aerial photography. The equipment can, however, also be used for measuring other lens types. Processing the electric signals is outlined schematically in Fig. 4. Any frequency scale corrections for off-axis measurements are taken into account in the electronics of the instrument. The equipment works very fast and the results displayed directly on the $X / Y$ plotter usually require no correction. In designing the equipment, great importance was placed on mechanical stability. The lens mount, fixed free from play, makes it possible to centre the test lens upon rotation. Fig. 5 shows a photograph of the equipment with

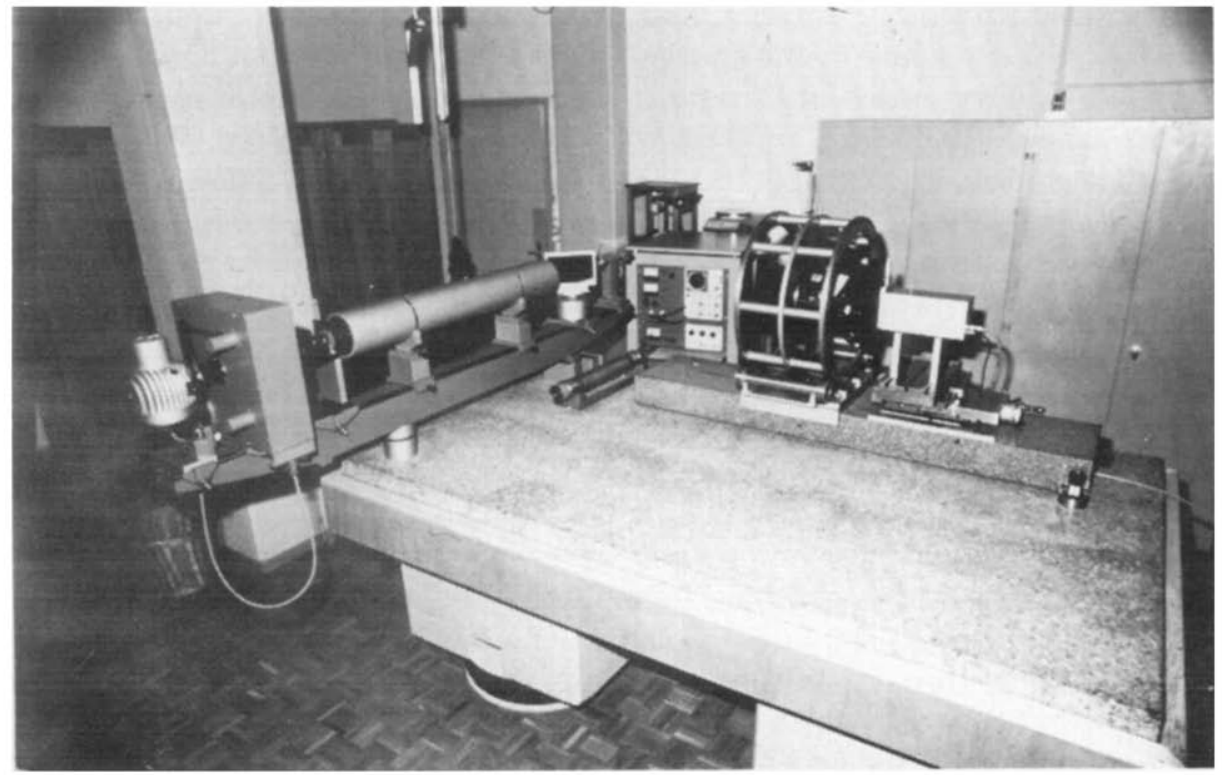

Fig. 5. View of the equipment used at Wild Heerbrugg. 
the test lens, as designed and constructed at Heerbrugg. A similar equipment was developed at the Institute of Applied Physics (Department of Industrial Research) of the Swiss Federal Institute of Technology, ETHZ, Zurich.

\section{PRECAUTIONS TO BE TAKEN INTO ACCOUNT WHEN CONDUCTING TEST MEASUREMENTS}

A few recommendations should be made on the basis of past experience with different comparison measurements. Firstly, a good measuring instrument is essential for reliable and accurate results. Secondly, the measurements must be taken under stipulated conditions (DIN recommendation). The conditions required for obtaining comparable results will now be summarised: Verification is carried out by means of the MTF measurement at high spatial frequency. Further care must be taken that nearly incoherent illumination is applied, and about the permissible size of the scanning range (range for isoplan conditions to be accomplished). Care must also be taken with the spectral frequency range as well as with the definition of the image position to be chosen. When a new test model is mounted, the lens is centred in the mount. Symmetry is verified by measuring in different azimuths by rotating the test lens. MTF measurements have frequently been carried out at Heerbrugg at the following angles: $w=0,0.5,0.7,0.85$ and $1 w_{\max }$ ( $w_{\max }=$ maximum field angle). Our image heights are accordingly: $y^{\prime}=0,0.41,0.62,0.81$ and $1\left(y^{\prime}=1\right.$, corresponding to the half diagonal $=152 \mathrm{~mm}$ ).

Up till now, interlaboratory comparison measurements of the MTF have been conducted with monochromatic light at the prescribed band width of the filter used (often $\lambda=546 \mathrm{~nm}$, half width $20 \mathrm{~nm}$ ). If "white light" is used for the MTF measurement, the spectral distribution of the light source, the filter and auxiliary optics and that of the receiver (or the overall spectral response) should be known and stated for comparison measurements. The question then arises which spectral range should be adopted when measuring lenses used for aerial photography. In practical aerial photography the spectral responses of the film material differ significantly; hence, it becomes difficult to specify a universal standard. Little is found on this subject in the literature. We at Wild have tentatively divided the spectral distribution selected for testing aerial lenses into two groups depending on use and on the basis of the commonly used Kodak emulsions:

(1) Lens for black-and-white and colour photography (Kodak Plus-X, Type 2402, and colour DF, Type So-397)

(2) Lens for IR and false-colour photographs (IR S/W, Type 2424 , false-colour, Type 2443)

This classification seems justified due to the fact that colour photography has become more important in recent years; further, the same lenses are often used for both black-and-white and colour photography (or for both IR and false-colour). In addition to the relative spectral sensitivity of Kodak Aerofilms, average daylight $\left(5500^{\circ} \mathrm{K}\right)$ and the relative spectral characteristics above sea 
level have been assumed. For our comparison measurements, which will be discussed below, a spectral distribution considered for the calculations and measurements is indicated in Fig.6. This choice allows the comparisons of the results with our previous measurements of the resolving power.

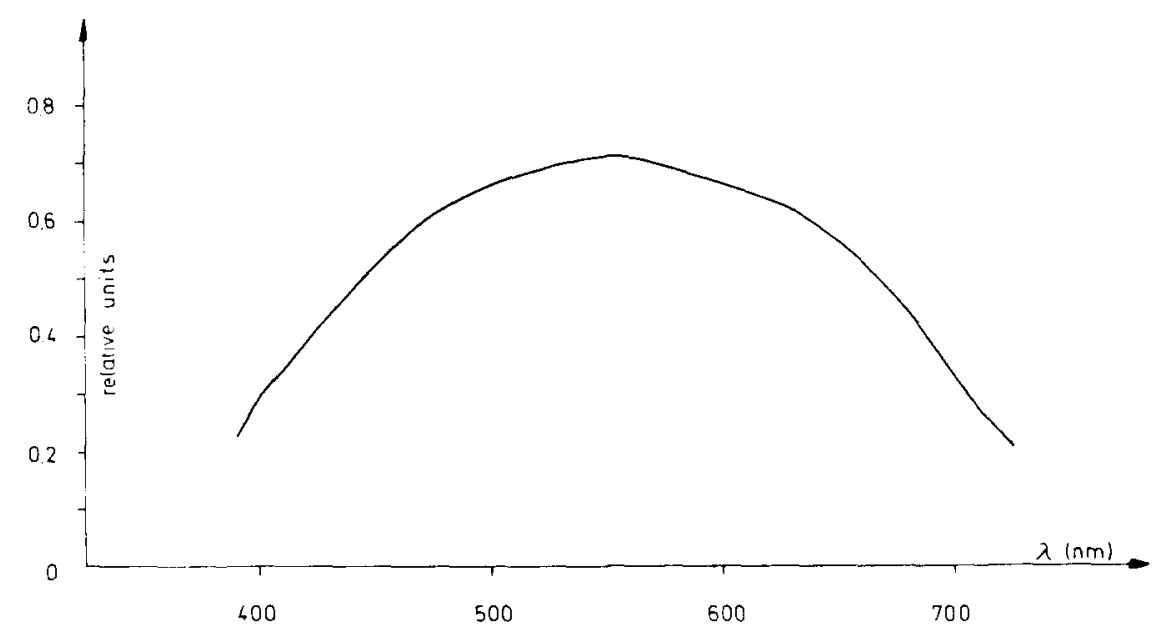

Fig. 6. Spectral distribution for measuring the MTF and for resolution test.

SOME REMARKS ON THE INFLUENCE OF CENTRING ERRORS ON THE MTF

Centring errors can be determined not only with the aid of the phase, but also by MTF measurements at different azimuths. Small symmetry errors in the optical system can change the MTF significantly (Beyeler and Tiziani, 1976). Studying the centring error of the optical systems by MTF measurements requires an investigation of the MTF in different azimuths for at least two field angles, preferably in the tangential direction. It was found that 0.5 and 0.7 of the maximum field angle are appropriate for symmetry investigations. MTF measurements are very sensitive to decentring.

It has been shown (Beyeler and Tiziani, 1976) that the influence of centring errors on the MTF can easily be computed using an approximate formula. To compare the theoretical results with actual measurements, experimental tests were set up at the Swiss Federal Institute of Technology in Zurich. For quantitative comparisons, the first lens of a Tessar System, with a relatively small sensitivity to decentring, was chosen and then decentred deliberately. Its influence on the OTF measurements is compared in Figs. 7 and 8 with computed results obtained by accurate ray-tracing techniques, as well as by means of the approximate formula for monochromatic light (Fig.7). The comparison of the results obtained shows that a very good agreement was 
achieved between calculated and measured results. The MTF is therefore very useful for detecting and measuring centring errors. For comparison, Fig. 8 shows the result of the same centring error on the phase measurement together with the calculated values (accurate and approximate). Additional results, off axis, are demonstrated in Beyeler and Tiziani (1976). The maximum permissible error depends on the application. For aerial-survey lenses the asymmetry of the MTF for two field angles and different azimuths was for our purpose supposed to be smaller than 0.10 .

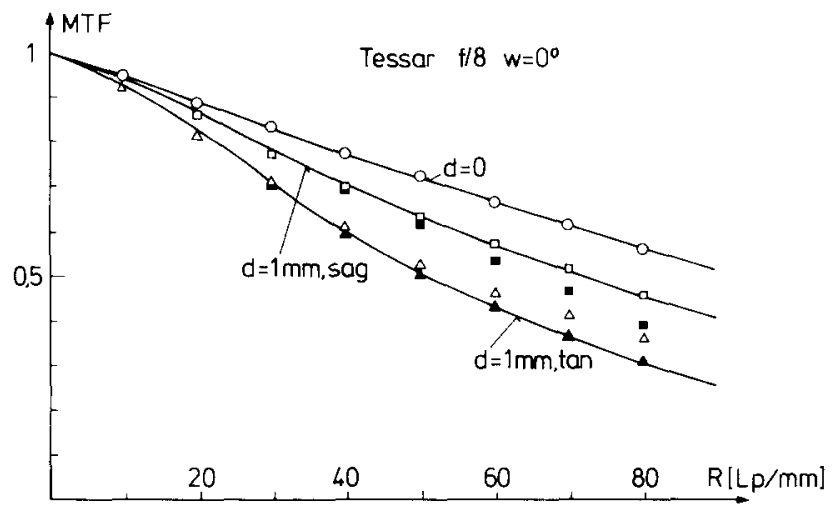

Fig. 7. MTF calculated and measured values of the deliberately decentred front lens of the Tessar objective on axis for $\lambda=543 \mathrm{~nm}$.

$\mathrm{O}=$ accurate calculated values for $d=0 ; 0, \square=$ accurate and approximate calculated values respectively for eccentricity $d=1 \mathrm{~mm}$ in sagittal direction; $\Delta, \Delta=$ accurate and approximate calculated values respectively for eccentricity $d=1 \mathrm{~mm}$ in tangential direction; $-=$ measured values.

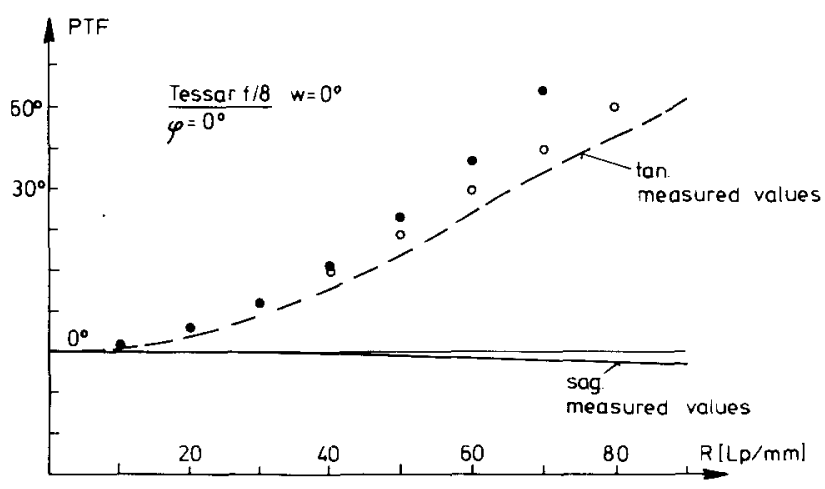

Fig. 8. Calculated and measured phase for the Tessar lens, on axis for $\lambda=543 \mathrm{~nm}$.

$0, \bullet=$ accurate and approximate calculated values, respectively;,$---=$ measured values. 
COMPARISON BETWEEN CALCULATING AND MEASURING THE MTF (mono and polychromatic)

To compare theoretical and measured values, wavelengths with band widths, aperture and focusing planes must be known exactly. The theoretical values derived from calculation must coincide with the measurement conditions. In the case of "white light" simulation, not only filters but also the spectral transmission of the lens, especially in the blue zone, must also be kept in mind. The absorption factor of the glass and the coating layers (single or multiple coatings) are mostly responsible for the variation in the spectral transmissivity of wide-angle lenses. Fig. 9 shows a typical transmission curve for a Universal Aviogon Lens (UAgI). This factor must be kept in mind, when weighting colours for comparison between calculated and measured values, especially considering the increased use of colour photography.

In computing the OTF all optical data of the system must be known. Our program for the calculation of the OTF was developed at the Institute of Applied Physics (Department of Industrial Research) of the Swiss Federal Institute of Technology, Zurich. It is based on the auto-correlation of the pupil function, for which the wave-front aberrations in the exit pupil must be known precisely. The program is optimized for accuracy $(\leqq 1 \%)$ in relation to computing time. The studies carried out have led to the conclusion that the exact shape of the vignetted pupil must be determined in order to calculate precisely the OTF with the help of auto-correlation, especially for large field angles. Approximations quickly led to large errors. To calculate the OTF for "white light", the OTF values weighted for the individual colours, as a function of the given spectral distribution, are vectorially added for the corresponding image angle. Details of the method, yet to be published, will be given in a future report. The question that immediately arises is, how the best

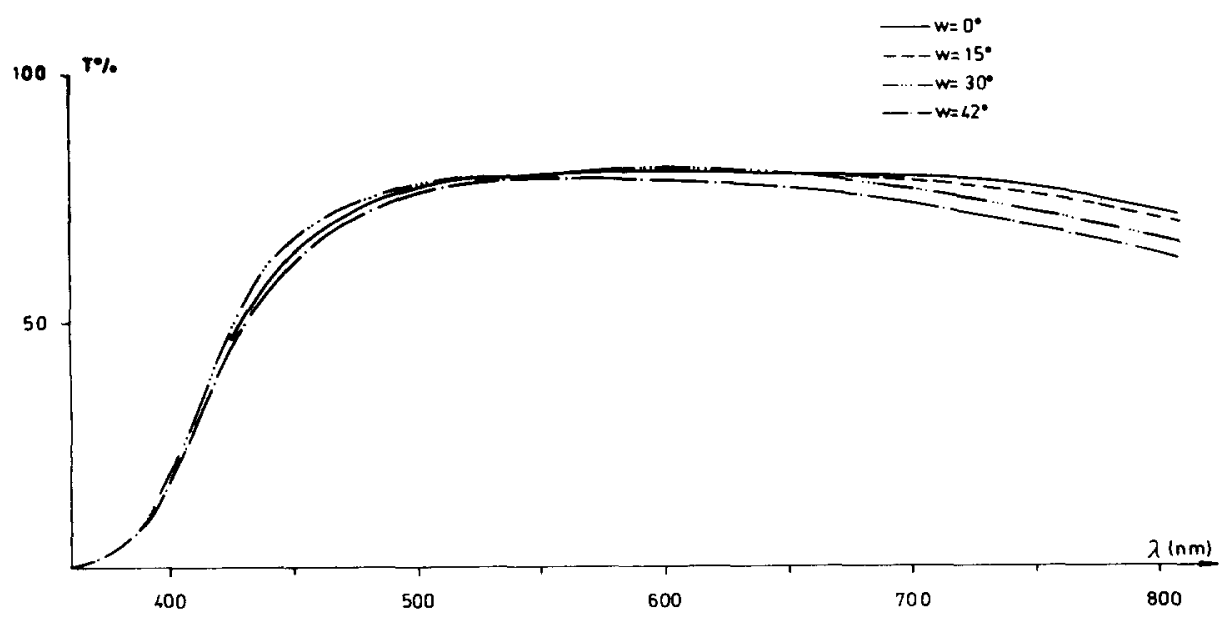

Fig. 9. Representative transmission curve for the Universal Aviogon Lens $(f=152 \mathrm{~mm})$. 
plane of reference can be found. The method we apply first is to focus by a fixed spatial frequency, determined to be $20 \mathrm{lp} / \mathrm{mm}$, for the individual image angle with "white light". With this method we can estimate the best image plane. Thus it becomes apparent that the curves for large image angles change only slightly upon focusing through. Finally, the best image position is optimized with a quality criterion, taking into consideration both the areas under the MTF curves and the regularity over the image field as will be discussed.

For comparison purposes, two objective types were selected, namely: the older $15 \mathrm{UAgI}(f=152 \mathrm{~mm})$ and the more recent $21 \mathrm{NAgII}(f=210 \mathrm{~mm})$ (Tiziani, 1975). Fig.10 shows a cross-section through the new Wild 21NAgII lens $f=210 \mathrm{~mm}, 1: 4$. Fig. 11 illustrates the defocusing curves of the same lens for various image angles; aperture $\mathrm{f} / 4, \lambda=588 \mathrm{~nm}$ and "white light" (spectral distribution as per Fig.6). In Fig. 11 the calculated values are marked with circles. The best image plane is usually slightly displaced, however, if the aperture is changed. Manufacturing is never $100 \%$ perfect, and minor residual imperfections in the lens components must always be expected. This leads, for example, to a change in the distance between the rear vertex of the last lens and the image; therefore, this distance is not suitable as a reference dimension. In addition, the focal length will change. Our studies indicate that the maximum value of MTF upon defocusing for given wavelengths (in this instance $\lambda=588 \mathrm{~nm}$ ) is useful as a reference measurement. Therefore, we try first to find the maximum value of the MTF on the axis, for example at $20 \mathrm{lp} / \mathrm{mm}$, and defocus to the best reference plane which has been optimized for the entire image field. $\Delta f=-0.12 \mathrm{~mm}, \pm 0.01 \mathrm{~mm}$ in our example was calculated theoretically and proved experimentally. Excellent agreement has been obtained up to now between the values calculated on the basis of optical data and those measured. For example, for a given image criterion and for the system studied, some of the quantitative comparison tests will be described. The deviations between the best image planes obtained from MTF calculation and measurement were $\Delta f \leqq 0.02 \mathrm{~mm}$ with an aperture of $f / 4$.

In Fig. 12 the monochromatic MTF values for three wavelengths, $\lambda=486$, 588 and $656 \mathrm{~nm}$ for the $21 \mathrm{NAgII}$ lens are plotted. In addition, the calculated phases are indicated. For this example, the largest deviation between calculated values and the results of the measurements carried out with monochromatic

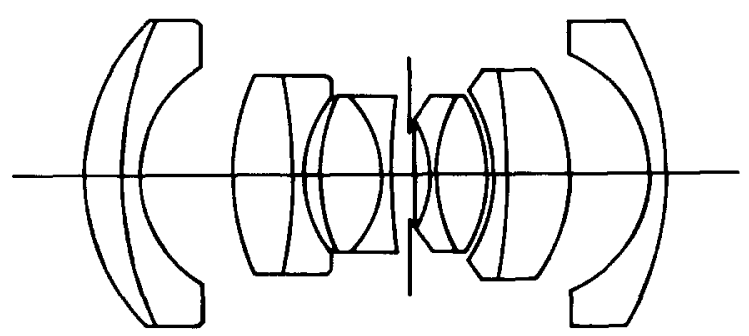

Fig. 10. Optical diagram of the new Wild 21 NAgII lens cone. 

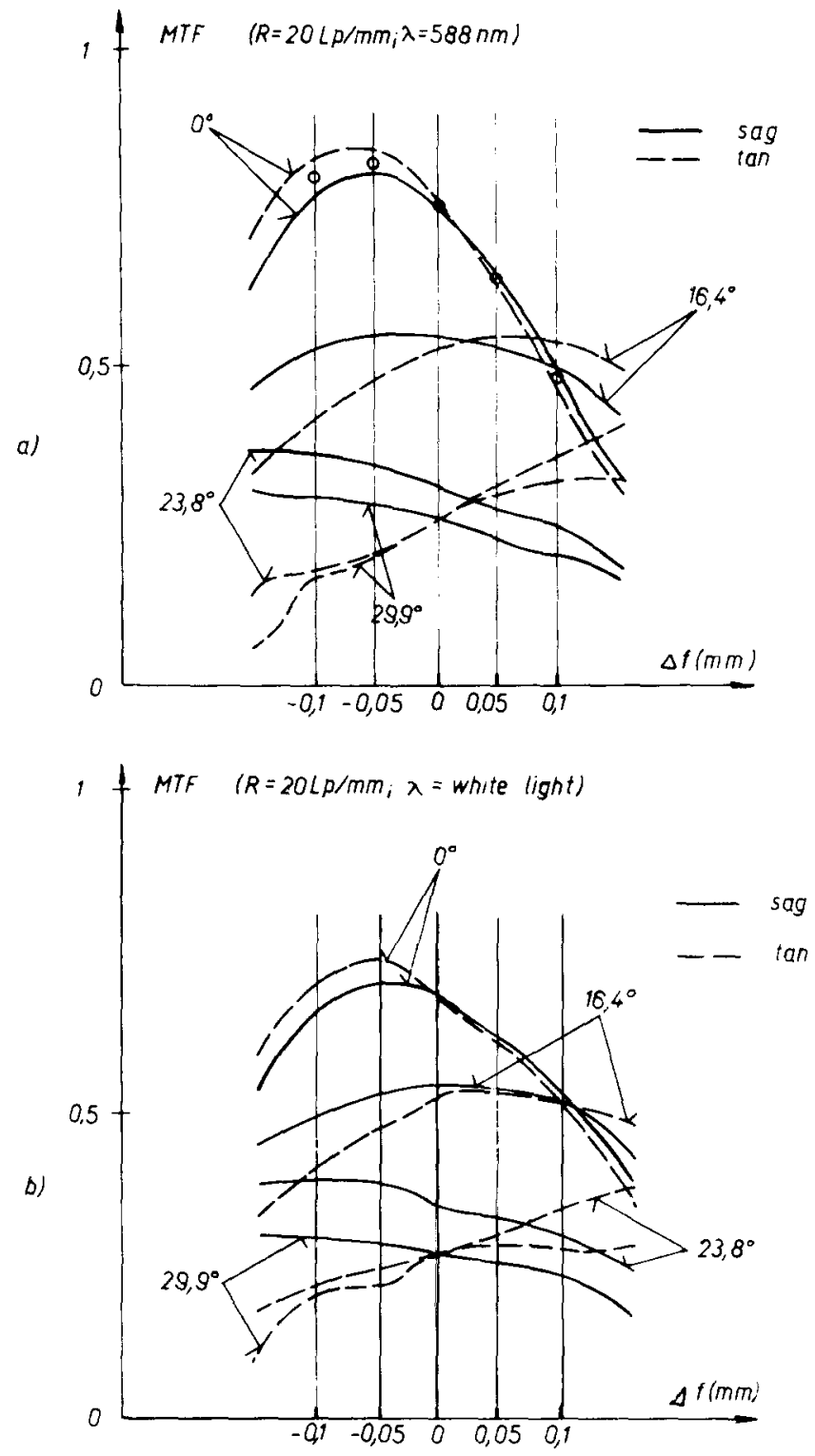

Fig.11. Variation of the MTF for different image angles at a fixed frequency of $20 \mathrm{lp} / \mathrm{mm}$ upon defocusing. a. For $\lambda=588 \mathrm{~nm}$. b. For "white light". $O O=$ calculated values

light was found to be $\leqq 0.07$, for $\lambda=486 \mathrm{~nm}$. In Fig.13 the results of the calculated and measured MTF with "white light" are reproduced. The measured values are marked with circles or crosses referring to sagittal or tangential section. Special attention should be paid to the relatively small phases for different spatial frequencies and field angles. It was also found that, for this lens, five wave lengths suffice to synthesize "white light" for the calculation. 

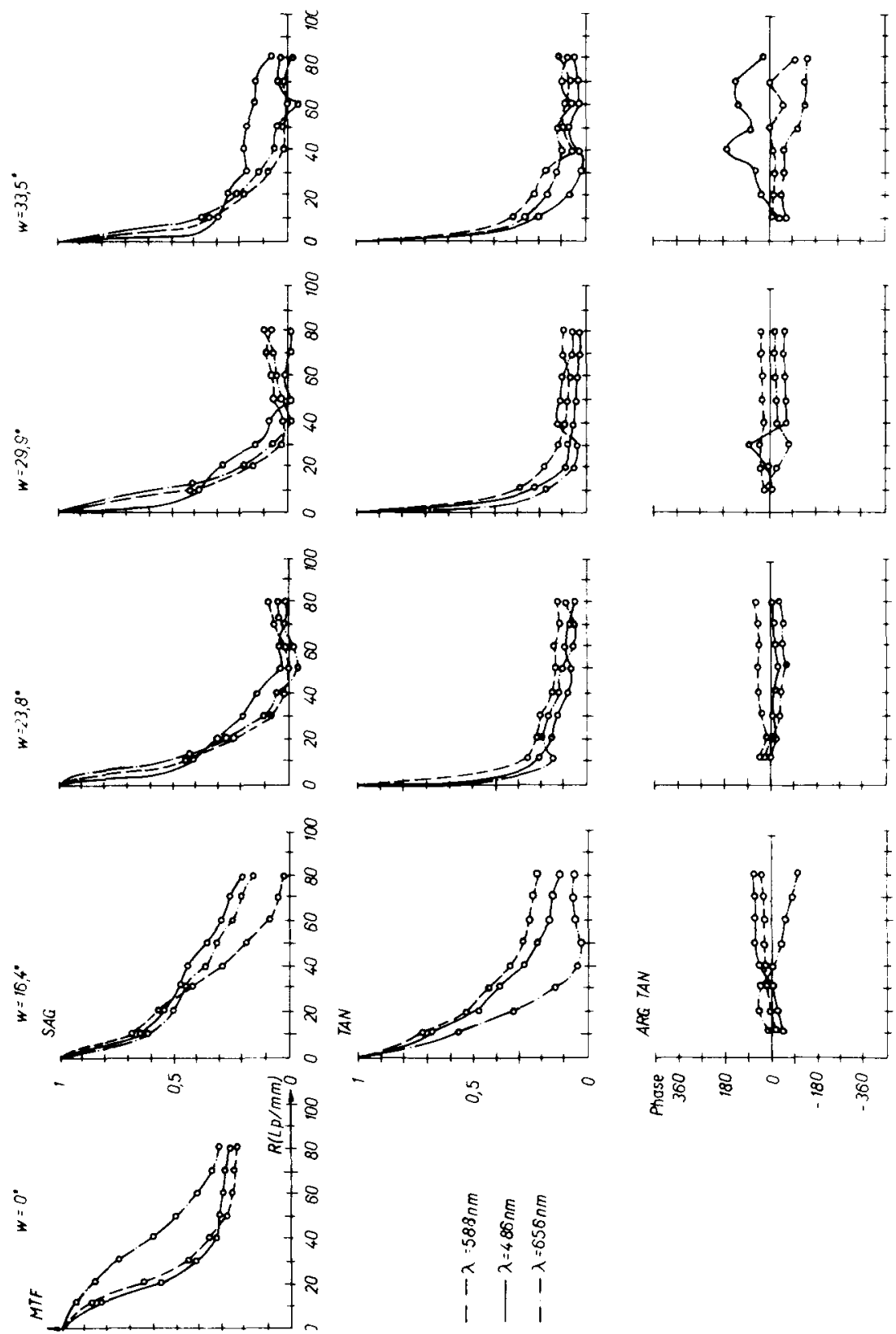

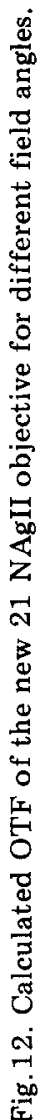




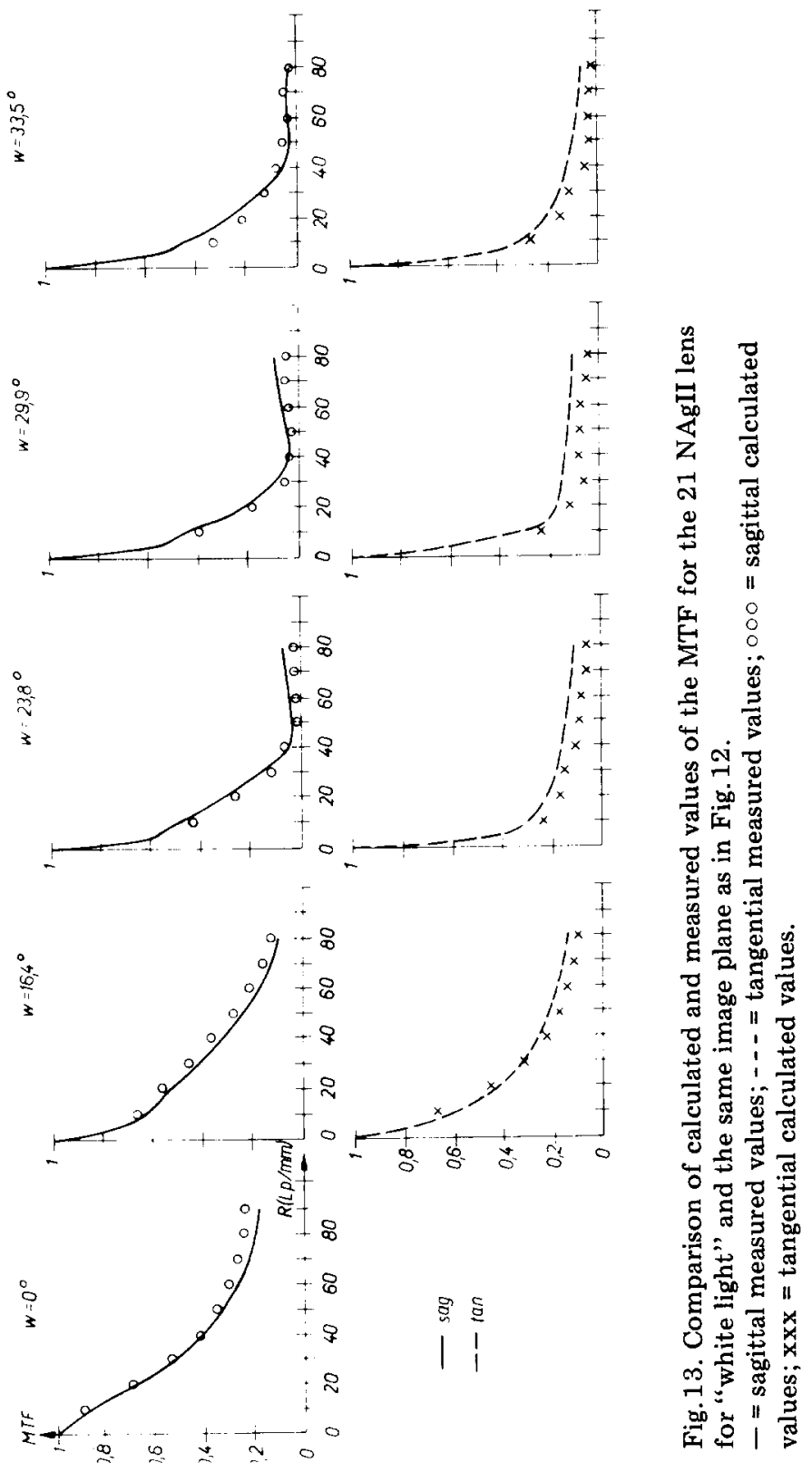


QUANTITATIVE COMPARISONS BETWEEN MODULATION TRANSFER FUNCTION AND RESOLUTION

Let us now compare the commonly used resolution test with the new test based on the MTF technique. For this purpose, MTF measurements and the classical power of resolution test were carried out. As many measurement conditions as possible, such as spectral distribution of the light source, maintaining identical image plane, should be fulfilled. In addition, it is necessary to reduce the subjective measurement errors. The contrast rendition of the data carrier (the photographic layer) must also be taken into account.

Until now, the visual resolving power on the image side has been used to determine the quality of the aerial photographic lens. Using a classical test pattern, the resolution is defined as the largest number of line pairs per millimeter which can still be discerned as separate lines in the projected image. For our comparisons, the object contrast was assumed to be one. The observer is, however, a subjective factor in the chain; the resolution of the human eye is dependent on the fixed frequency, on the contrast of the image, and on the brightness of the image, as it is presented to the eye. Errors arising through the observer's subjective viewing may be reduced if the resolution of the image (in our case a three-bar test plate) is determined with the help of a microscope until the optimum reproducible conditions for observation (magnification, illumination) have been attained. In addition, our resolution tests were conducted by well-experienced personnel.

Threshold curves have been proposed by different authors (Brock et al., 1972). For quantitative results, examination of several types of lenses with numerous observers led to the conclusions that, for our conditions, the contrast threshold of the eye may be assumed to be $C_{\mathrm{A}}=0.03$ for a well-trained observer. On the basis of the $\mathrm{MTF}_{\mathrm{E}}$ of the emulsion used (Kodak Plus-X and Agfapan 25) and that of the microscope used (M5) $\left(\mathrm{MTF}_{M}\right)$, a threshold value SW was obtained (Fig. 14) whereby:

$\mathrm{SW}=\frac{C_{\mathrm{A}}}{\mathrm{MTF}_{\mathrm{E}} \cdot \mathrm{MTF}_{\mathrm{M}}}$

The microscope is mostly used to reduce the influence of the observer on the results, otherwise the MTF of the eye would have to be introduced. Illumination and magnification can be adapted to obtain the best condition to analyse the image on the appropriate emulsion. The microscope can be selected such that its $\mathrm{MTF}_{\mathrm{M}} \approx 1$ for the frequency range $0<R<60 \mathrm{lp} / \mathrm{mm}$. This was, however, not the case for our investigations. Coherence problems do not occur, when the developed image of the three-bar target is measured.

The point where the threshold curve intersects the MTF curve of the lens is the point of its resolution. The plot of the MTF curve indicates however more information about the quality of the lens performance than the resolution test alone. 


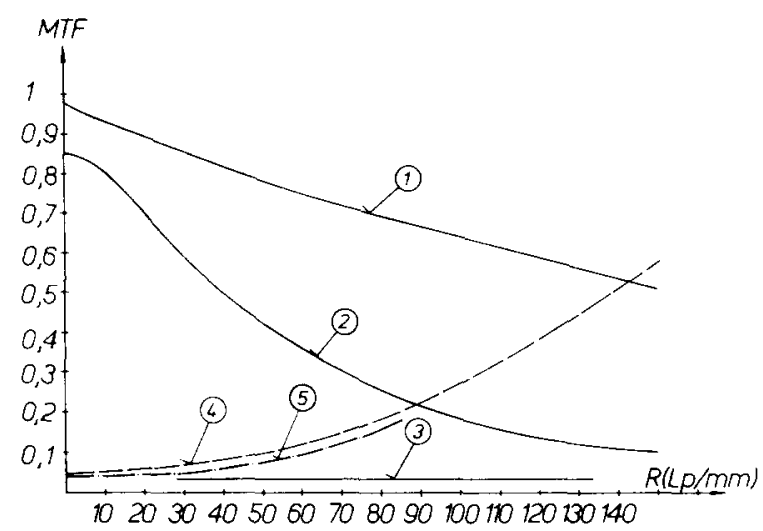

Fig.14. Determination of the threshold curve. $1=\mathrm{MTF}_{\mathrm{M}}$ of the microscope; $2=\mathrm{MTF}_{\mathrm{E}}$ of the emulsion (Kodak $+\mathrm{X}$ ); 3 = threshold of the human eye; $4=$ threshold for Kodak + $\mathrm{X}$-emulsion; 5 = threshold for Agfapan 25.

A quantitative comparison between the MTF measurement and the resolution test was undertaken. By observing the above-mentioned precautions, good agreement was obtained between the subjective resolution tests and tests made with the help of the MTF and the threshold-value curve. In order to compare the results of previous resolution tests with the new, more rapid and more objective methods, it was expedient to adjust the spectral distribution of the measurements to those of Fig. 6.

The MTF curve for simulated "white light", using the new Wild objective, 21 NAgII ( $f=210 \mathrm{~mm}, f / 4)$, is shown for various image angles in Fig. 15 . Fig. 16 shows those for an older Wild Aviogon 15 UAgI lens $(f=152 \mathrm{~mm}$, $f / 5.6)$. For these lenses good agreement was obtained between the calculated and measured values (monochromatic and polychromatic). The threshold curves are also entered on these graphs. It should be pointed out that with the older 15 UAgI lens pseudoresolution can occur for some spatial frequencies. To ensure agreement of the identical image planes for visual resolution tests and MTF measurements, the position of the pressure frame (image plane) was accurately measured. Consequently, it appeared that the best image plane, which required a great deal of work to find by recording different photographs in different focusing positions, lay near the one determined by means of MTF measurements. It seems that MTF measurements are faster and more reliable than classical techniques for determining the best image position.

Figs. 17a and 17b show a comparison between the resolution found with the MTF and threshold value curves (indicated with circles for sagittal and crosses for tangential direction) and the results of subjective resolution tests (again in tangential and sagittal directions). The examples presented were conducted with object contrast one. However, MTF measurements can easily be converted for object contrast $\neq 1$. 

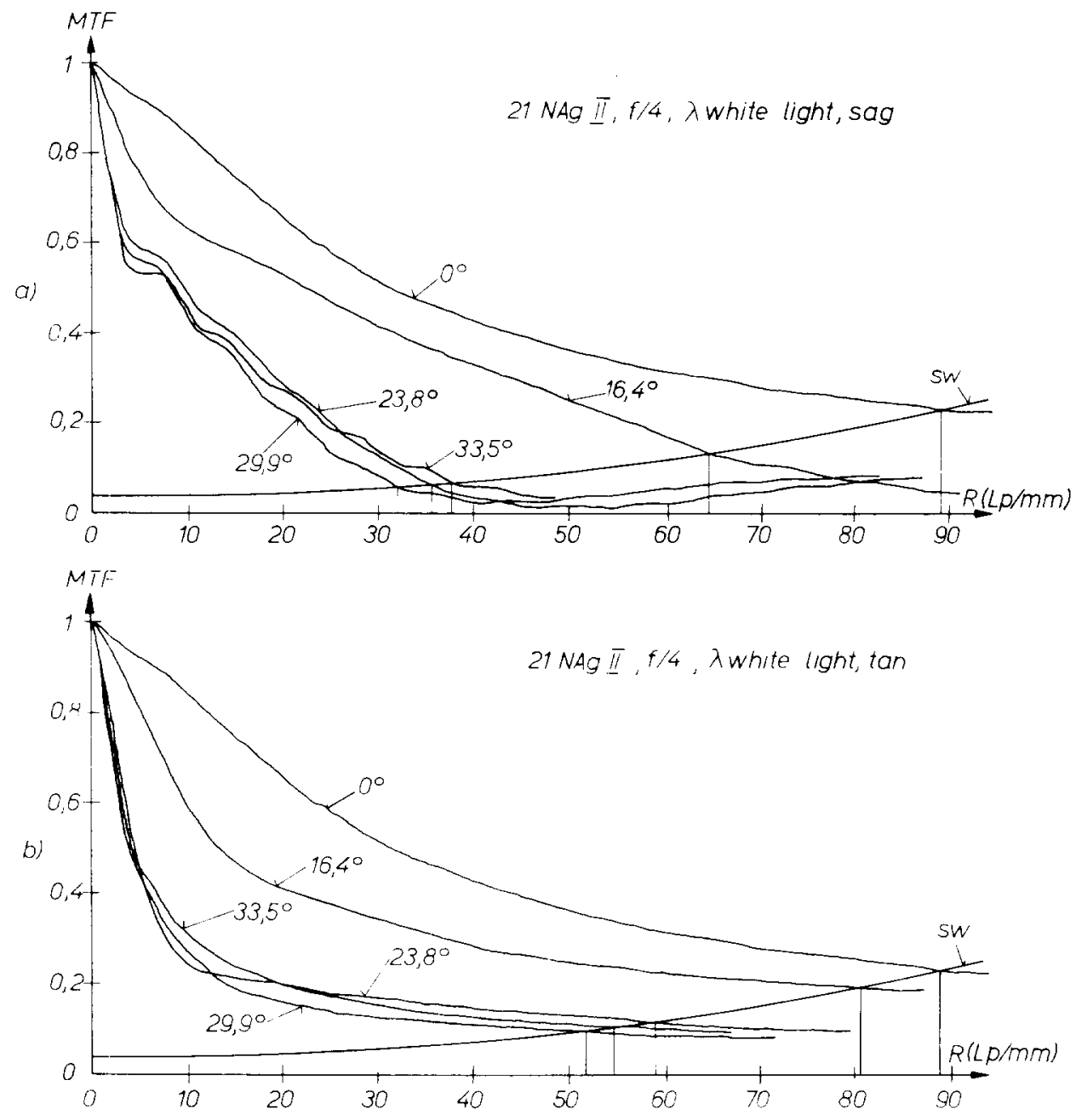

Fig. 15. MTF curve of the 21 NAgII lens $f / 4$ for "white light" and threshold SW; $w=0^{\circ}$, $16.4^{\circ}, 23.8^{\circ}, 29.9^{\circ}$ and $33.5^{\circ}$. a. Sagittal. b. Tangential.

\section{REMARKS CONCERNING IMAGE-QUALITY CRITERIA}

Quality criteria are valuable for finding the best image plane and for comparing the quality of various optical systems. Practical, useful imagequality criteria necessitate, however, reducing the accumulating MTF data to a minimum. Linfoot (1960) and Hertel (1963) began studying image-quality criteria some time ago, but up to now their results have not been put to practical use. For many optical systems, image-quality criteria based on OTF are not sufficient and additional criteria such as distortion and lateral chromatic aberrations need to be considered. Hence, image-quality criteria 

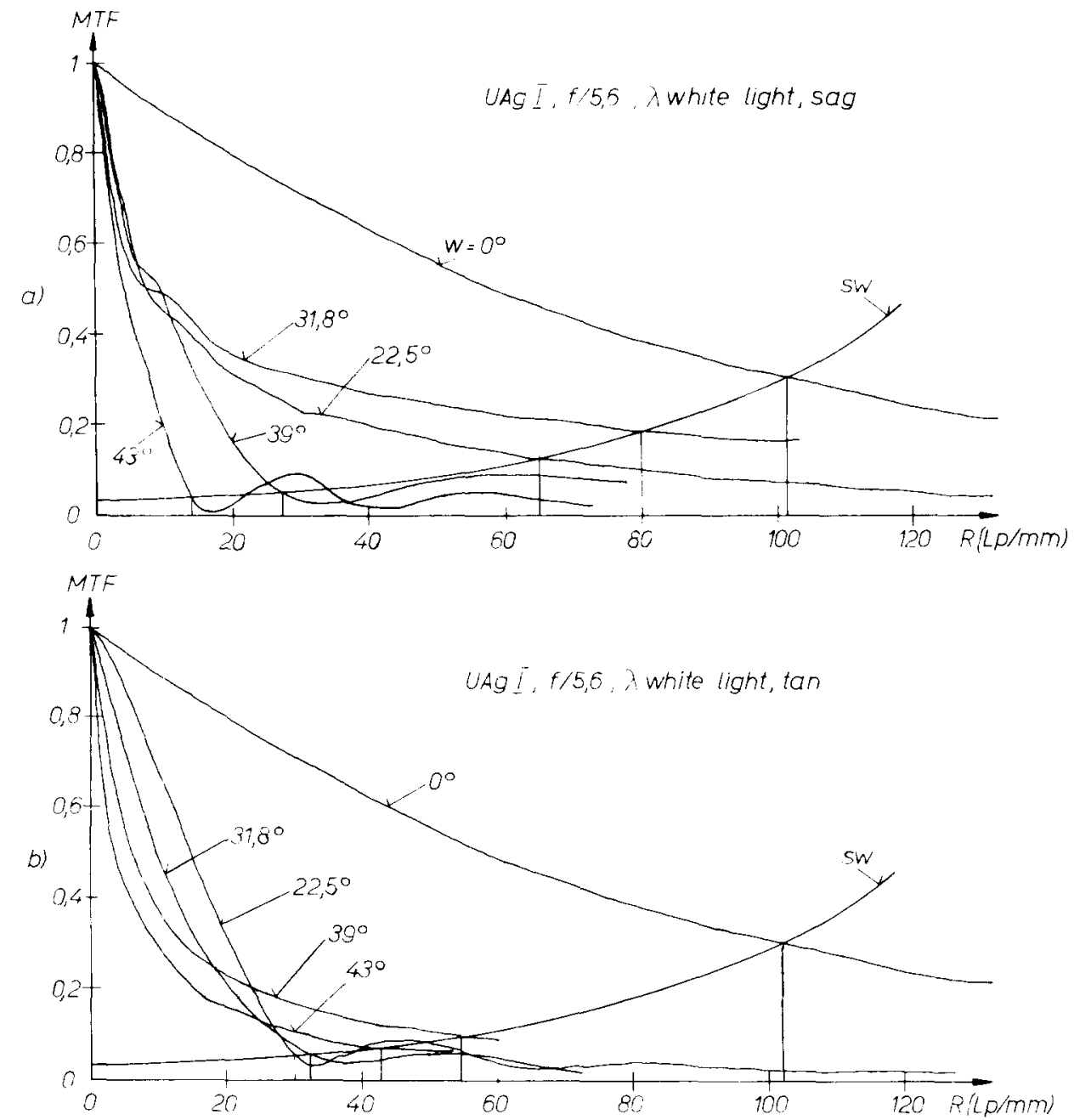

Fig. 16. MTF curves of the older Wild 15 UAgI lens $f / 5.6(f=152 \mathrm{~mm})$ for "white light" and threshold value SW; $w=0^{\circ}, 22.5^{\circ}, 31.8^{\circ}, 39^{\circ}$ and $43^{\circ}$. a. Sagittal. b. Tangential.

for aerial survey lenses based on OTF are not the same as for photographic systems of other kinds, e.g. amateur photography. At present, in order to evaluate picture quality in photogrammetry, an average value of the resolution over the entire picture format is determined for the given image planes (AWAR). Thereby the resolution angle for every test for a given field angle is multiplied by the following ratio: size of zone in which image point is located to size of entire format. The weighted resolutions for the different field angles are added to obtain the AWAR (average weighted areal resolution). Only one value, the geometric mean from the tangential and sagittal resolution for every test angle, is used for this purpose. 
The image-quality criteria can be considerably expanded if the weighted areas under the MTF curves are applied instead of the resolution. To compare the image quality of various lenses when using MTF as a quality criterion, or to find the best image plane, the number of curves must be reduced. To
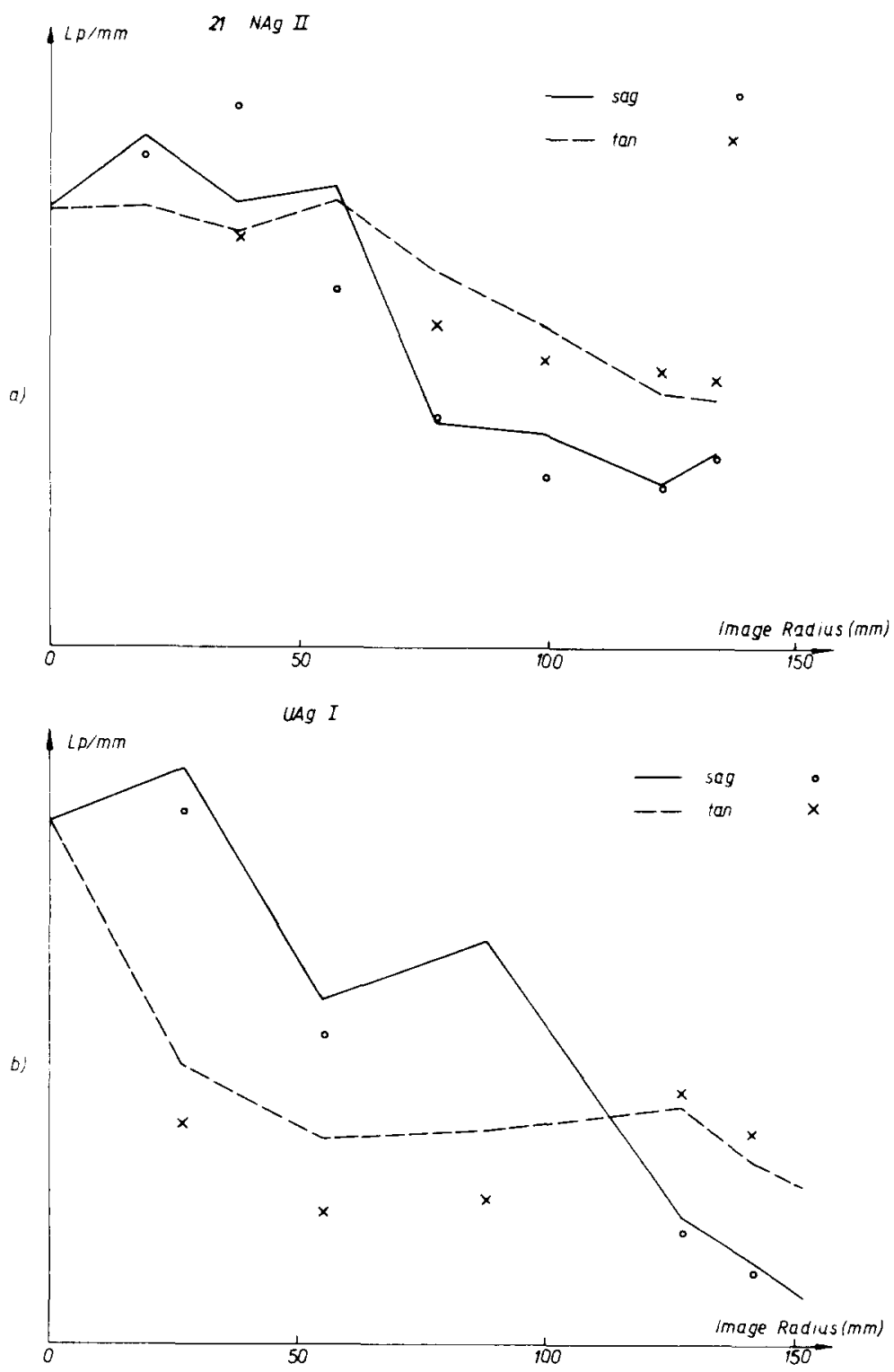

Fig.17. Resolutions of the Wild 21 NAgII, $f / 4$ (a) and Wild 15 UAgI, $f / 5.6$ (b), for "white light", plotted as a function of the image radius. Sagittal: $-=$ conventional resolution test; $000=$ with help of the MTF.

Tangential : - - = conventional resolution test; $\mathbf{x x x}=$ with help of the MTF. 
evaluate lenses on a production basis, one might even go so far as to use a few pre-selected spatial frequencies. In addition, the users of image-quality criteria also have various needs. For production tests only a few curves are required. If a new lens is being tested, the various MTF curves are extremely instructive for the optical designer. For this purpose a great deal of information for various apertures, colours and image planes is needed.

Image-quality criteria based on MTF for determining the best image plane and for evaluating and comparing different lenses will now be proposed. Three points should be taken into consideration:

(1) The area under the MTF curve up to the threshold value.

(2) Regularity with respect to tangential and sagittal MTF.

(3) Regularity over the entire image field.

Different possibilities for this task were considered. The most promising approach found so far for the systems studied will be described briefly. The area under the MTF curve is integrated up to the threshold curve. The geometric mean of the sagittal $\left(\mathrm{MTF}_{\mathrm{s}}\right)$ and tangential MTF $\left(\mathrm{MTF}_{\mathrm{t}}\right)$ is selected in order to reduce the number of curves, hence

$T_{\mathrm{g}}=\sqrt{\mathrm{MTF}_{\mathrm{s}} \cdot \mathrm{MTF}_{\mathrm{t}}}$

To take into account the difference between $\mathrm{MTF}_{\mathrm{s}}$ and $\mathrm{MTF}_{\mathrm{t}}$ at the same field angle, the point of intersection of the poorer MTF curve with the treshold-value curve is adopted as the limiting frequency for the integration. For the same $T_{\mathrm{g}}, \mathrm{MTF}_{\mathrm{t}}$ mat be very poor and $\mathrm{MTF}_{\mathrm{s}}$ very good. But $\mathrm{MTF}_{\mathrm{t}} \approx$ $\mathrm{MTF}_{\mathrm{s}}$ would be preferable for most applications. In the case of curves which vary too much, therefore, the surfaces used for evaluating image quality are smaller. For information about the image quality over the entire image field, the area under the $T_{\mathrm{g}}$ curve is weighted with the surface weight $p_{i}$ of the image point concerned (similar to the AWAR). Thus $p_{\mathrm{i}}$ takes into account the relationship of that part belonging to image angle $w_{i}$ with respect to the entire image surface. To assess the regularity over the image field, the harmonic mean is carried out and presented in addition to the arithmetic mean. In Fig. 18 consideration is made for both the arithmetic and harmonic mean of the weighted areas under the $T_{\mathrm{g}}$ curves, $Q_{i}$ for the different field angles. Furthermore, it was found that the structural content proposed by Linfoot (1960) changes the plot of the curve only slightly. Typical results of the 21 NAgII lens for $\lambda=588 \mathrm{~nm}$ and white light are illustrated in Figs. 18a and 18b.

In these Figures the curve (1) illustrates the arithmetic mean over the image field, defined as:

$$
A_{1}=\frac{\sum_{i=1}^{N} p_{i} Q_{i}}{\sum_{i=1}^{N} p_{i}}
$$


with:

$$
Q_{i}=\int_{0}^{R_{g}} T_{i_{g}}(R) \mathrm{d} R
$$

and:

$$
\begin{aligned}
T_{\mathrm{g}}=\sqrt{\mathrm{MTF}_{\mathrm{s}} \cdot \mathrm{MTF}_{\mathrm{t}}} \\
\text { where } p_{i}=\text { surface weights, } \\
i=1,2, \ldots, N, \\
N=\text { number of field angles, and } \\
R_{\mathrm{g}}=\text { limiting frequency. }
\end{aligned}
$$

The curve (2) shows the harmonic mean, each time, which allows for the regularity over the entire image field:

$$
A_{2}=\frac{\sum_{i=1}^{N} \frac{\frac{1}{p_{i}}}{Q_{i}}}{\sum_{i=1}^{N} p_{i}}
$$

In curve (3) $Q_{i}$ is replaced by:

$\overline{Q_{i}}=\int_{0}^{R_{\mathrm{g}}}\left|T_{i_{\mathrm{g}}}(R)\right|^{2} \mathrm{~d} R$

which takes into consideration the structural contents proposed by Linfoot (1960). Curve (3) in the examples investigated so far does not appear to produce any results essentially different from those produced by curve (1), even though its calculation is more time consuming for practical purposes. The theoretical values obtained from the calculated curves are designated with circles. Our studies have shown that in taking into account the regularity over the image field - i.e. using the harmonic mean - the best image plane is shifted slightly relative to that of the arithmetic mean. The distance between the maximum of the arithmetic and harmonic mean gives an indication of the homogeneity over the field. For many applications it seems to be best to choose the image position between the two maxima for a given aperture of the lens.

In conclusion it may be said that image quality can be assessed with objective criteria. Some work remains to be done and additional comparison measurements still have to be carried out in order to verify the quality figures proposed in this paper. They apply basically for the lenses, but could be expanded to be used for the whole system. 


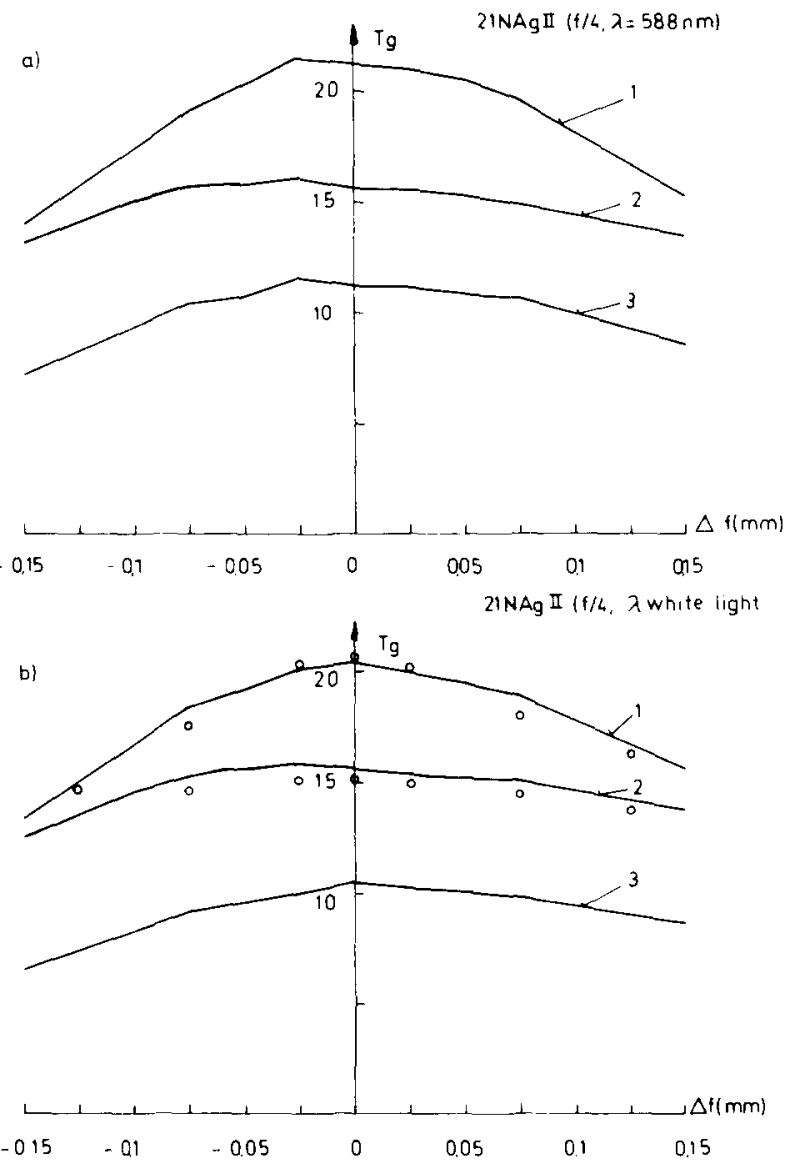

Fig. 18. Quality criteria averaged over the entire image, as a function of defocusing, for $\lambda=588 \mathrm{~nm}(\mathrm{a})$ and for white light (b). $1=$ Arithmetic mean; $2=$ harmonic mean; $3=$ structural content.

(The corresponding calculated results are shown with circles.)

\section{ACKNOWLEDGEMENT}

I should like to express my thanks to Mr. A. Aemmer of the Applied Physics Department of the Swiss Federal Institute of Technology for carrying out most of the computations and to Mr. J. Behringer for measurements.

\section{REFERENCES}

Baker, L.R., 1971. Status of OTF in 1970. Opt. Acta, 18(2): 81-92.

Beyeler, B. and Tiziani, H., 1976. Die optische Übertragungsfunktion von dezentrierten optischen Systemen. Optik, 44(3): 317-328. 
British Standard BS 4779, 1971. Recommendations for the Measurement of the Optical Transfer. British Standards Institution, London.

Brock, G.C., Marchant, A.C. and Williams, T.L.W., 1972. OTF standards for aerial mapping lenses. Opt. Acta, 19(12): 953-972.

Consitt, F.J.P. and Mandler, W., 1971. OTF techniques in routine testing of production lenses. Opt. Acta, 18(2): 123-131.

DIN 58185. Optical Transfer Function. German Standards Committee, Pforzheim.

Hertel, J., 1963. Mitteilungen und Berichte des optischen Instituts der TU Berlin 7.

Hopkins, R.E. and Dutton, D., 1971. MTF measurements and computations on a large wide-angle lens. Opt. Acta, 18(2): 105-121.

Kuttner, P., 1975. Interlaboratory comparisons of MTF measurements. Opt. Acta, 22(4): $265-75$.

Linfoot, E.H., 1960. Qualitätsbewertung optischer Bilder. Vieweg \& Sohn, Braunschweig.

Matsui, Y. and Murata, K., 1971. Comparison test of several OTF measuring equipments. Opt. Acta, 18(2): 149-163.

Murata, K., 1966. In: E. Wolf (Editor), Progress in Optics, 5. North-Holland, Amsterdam, pp. $199-245$.

Norton, C.L., 1975. Optical and modulation transfer functions. Photogramm. Eng. Remote Sens., 41(2): 203-216.

Pouleau, J. and Lafouasse, P., 1972. La mésure de la F.T.M. par l'ACOFAM-Matra et la généralisation d'une méthode d'évaluation des systèmes optiques.

Tiziani, H., 1975. Beurteilung der Bildqualität mit Hilfe der optischen Übertragungsfunktion. Festschrift Dr. h.c. L. Bertele zum 75. Geburtstag, Wild Heerbrugg, pp. 59-71.

Welch, R., 1974. Photogrammetric image evaluation techniques. ISP, Comm. I, Symposium, Stockholm. 